\title{
SMART SAFETY SYSTEMS: ARE THEY READY TO CONTROL THE HAZARD OF MAJOR ACCIDENTS?
}

\author{
SILVIA M. ANSALDI, PATRIZIA AGNELLO \& PAOLO A. BRAGATTO, \\ INAIL - DIT Centro Ricerca, via Fontana Candida, Rome, Italy
}

\begin{abstract}
In recent years, technological research have developed many innovative systems, aiming at exploiting the potential of "smart" technologies for the industrial and occupational safety. Many innovative smart systems are now coming to the market, promising major improvements at a very low cost. Decision makers are puzzled by the numbers and diversity of proposals. The choice is more difficult for the establishments featuring the hazard of major accidents, where the operators have to satisfy the requirements of the Seveso Directive. They have to evaluate if the proposed systems are ready to be used, if they are relevant for the control of major hazards, and if they are effective. The goal of the paper is to provide the decision makers with a tool to select systems suitable to be effectively appliedinto the industrial plants.
\end{abstract}

Keywords: smart safety systems, Industry 4.0; major accident hazard.

\section{INTRODUCTION}

In the last years, the manufacturing industry is rapidly changing thanks to some enabling technologies, including smart sensors, wearable computing, wireless communication, internet of things, artificial intelligence, cloud computing, augmented reality, robots and other autonomous intelligent systems. These technologies enable the gathering of huge amounts of data at every points of the process flow, and the fast communication between technical systems and organization at any level in the production chain. The potential of these systems is widely recognized, and it is referred as fourth industrial revolution or "Industry 4.0". The impact of Industry 4.0 on the industrial and occupational safety has not yet been adequately investigated. The focus of the present paper is on the potential of smart systems technologies for improving the control of major accident hazard, due to the use of hazardous materials in different industries (energy, oil and gas, chemical, transport, manufacturing). Despite occupational incidents, major industrial accidents affect, in addition to workers, to industrial assets, environment and external population. The prevention of industrial accident is regulated all over Europe by Seveso III Directive, which requires a stricter control of industrial establishment by control bodies. The scope of the paper is the "smart systems" that deal with the prevention of major accidents. Occupational safety partially overlaps the control of major accident hazard, thus also a number of systems for occupational safety have been included.

\section{OBJECTIVES}

"Smart systems" are defined as objects able to exchange information on themselves and on the surrounding area thanks to specific enabling communication technologies. For the purpose of the paper, the definition adopted for "smart system" is a system operating in low demand mode, which include functions of sensing, actuation, and control for describing and analysing a situation and, possibly, take decisions. The paper exclusively focuses on the Smart Systems used for Safety SSS purposes.

The paradigm "Internet of Things" IoT refers to a large number of "smart systems" talking to each other, in order that in living and working environment objects (e.g. machines, tools, clothing) are wireless connected between them, for making human activities easier and more 
effective. In many industries, such as manufacturing and construction, the IoT is already widely adopted to improve all the production activities, including quality and safety. Furthermore, the new safety technologies are valuable to fill in the gap between technical systems and safety procedures, as discussed by [1].

\subsection{Smart systems for safety in seveso directives establishments}

The Seveso Directive requires to Seveso establishments to run a quantitative risk analysis for identifying possible accidents and implementing adequate technical, procedural, and organizational measures to reduce as far as possible the likelihood of event and to mitigate its consequences.

The main scope of this research is to understand how much the adoption of SSS in a Seveso establishment may have impact on the quantitative risk analysis, by reducing the probability or by improving the barriers for mitigating the consequences of accidents involving hazardous materials.

The main users of the results of this research are the operators of the Seveso establishment. They need a sound scientific support to address the safety improvement and to select the SSS suitable for accident prevention. Results are useful also for control bodies, which are in charge to evaluate the adequateness of adopted measure for the risk reduction purpose. Afterwards, regulators may use the results for promoting risk reduction throughout the process industries, while the providers of smart systems may improve their offer by supplying Seveso establishments with specific safety solutions.

\section{METHODS FOR SSS EVALUATION}

In order to evaluate the impact of adopting SSS for reducing risks or for mitigating the consequences, some basic criteria have been identified, including: Readiness, Relevance, and Effectiveness. Each criterion settles four levels associated to increasing order rates, from the poor to the high value.

All the criteria have in common their relationship with respect to the Safety Management System SMS, which are considered as the engine for planning, working, checking, and managing safety in the establishments.

\subsection{Readiness criteria}

This criterion takes into account the readiness of twofold point of view, one from the technological and commercial availability of the SSS solutions and the other from the capacity of the organizations to adopt them inside their Safety Management System.

For the technological readiness, three levels have been considered. The lower level is the demonstration and validation at a pilot system. Conceptual and laboratory demonstrations are not included at all. The highest level is the innovative commercial product, which has been designed, produced and tested according to recognized engineering practice. At the intermediate level, there is the industrial prototype, which still needs a wide testing phase.

In spite of the fact that SMS is mandatory in Seveso establishment, the SMS capabilities vary by the organizations. Indeed, they may be at the low level, only a collection of basic procedures. At the intermediate level there are the systems featuring the compliance (and possibly the certification) with recognized safety standards. The highest-level deals with the adoption of a "smart" SMS that would be able to incorporate information coming by big data monitored through the SSS. This implies a SMS deeply renewal, moving from a traditional safety document management system towards a safety information and knowledge 
Table 1: Level of readiness.

\begin{tabular}{|l|l|}
\hline Level & Requirements \\
\hline 1 & $\begin{array}{l}\text { Experimental SSS \& Certified SMS } \\
\text { Prototype SSS \& Basic SMS }\end{array}$ \\
\hline 2 & $\begin{array}{l}\text { Experimental SSS \& Smart SMS } \\
\text { Prototype SSS \& Certified SMS } \\
\text { Commercial SSS \& Basic SMS }\end{array}$ \\
\hline 3 & $\begin{array}{l}\text { Prototype SSS \& Smart SMS } \\
\text { Commercial SSS \& Certified SMS }\end{array}$ \\
\hline 4 & Commercial SSS \& Smart SMS \\
\hline
\end{tabular}

management system. There are not yet standard definitions for "smart" SMS, but the "risk based" systems, which are adopted in industry for specific purpose (e.g., inspection or maintenance), are good examples of "smart systems". They are built on a sound knowledge of complex physical and chemical mechanism, continuously tuned through the gathered data and the managed information, according to a Bayesian approach. At the end, a SMS is considered "smart", if it has the capacity of collecting and discriminating huge amounts of data and upgrade the data into information, possibly assisted by artificial intelligence technologies.

The Table 1 illustrates the levels from combining those two factors, the SSS technological levels, and the SMS capabilities. It is important to outline that the SSS conceptual formulation is relevant only for evaluating the SSS technology readiness itself, while is meaningless by comparing with all levels of SMS capabilities. In the meanwhile, the introduction of SSS prototypes (level 2, only experimented in the lab) has not significance if SMS is only a basic and unstructured collection of safety documents.

The level 1 of Table 1 refers to SSS technologies experimented in pilot plants and included into traditional SMS in compliance with safety standards. It also corresponds to the case of introducing some SSS prototypes, already validated in relevant environment, into a basic SMS.

The level 2 corresponds to one of the following combinations:

- The SSS technologies are still in the experimental prototypes, but the SMS is able to take advantages of gathering those data, filtering their reliability.

- The SMS, in compliance with safety standards or officially certified, introduce SSS technologies, already validated in appropriate environments.

- The introduction of commercial SSS, or at least those smart systems with proven reliability factors, into basic SMS, which can evaluate them as traditional safety barriers.

The level 3 deals with one of the following factors:

- The SSS technologies, evaluated in meaningful environment, become part of a smart SMS, which is able to gather the data and discriminate their relevance and reliability.

- The introduction of commercial SSS or advanced prototypes into the traditional SMS.

Finally, the level 4 foresees the use of fully tested SSS technologies into smart SMS. To achieve such a result, the SMS needs to be dramatically improved, changing the approach of 
managing only safety documents into a more informatics management approach that can take advantages of both the applications used and the information developed. However, since a fully smart SMS is still not defined, the level 4 considered here indicates that the outcomes from those SSS are taken into account during specific activities and operations, e.g., maintenance, control.

\subsection{Relevance criteria}

The Smart Systems for Safety provide solutions with aiming more data on critical items, giving early warnings, improving workers behaviour and awareness, and so on. All these issues should deal with the Safety Management System, so that the SSS should strengthen resources, procedures and instructions included within the SMS.

The level, illustrated in Table 2, should point out how the system is specific for safety, and especially is closely appropriate to major accident hazards. It also takes into account if the smart system is able to control a specific risk or is a general type with safety implications.

The level 1 deals with smart systems that can avoid minor incidents, which could be concurrent or indirect cause of major accidents. The SSS at this level increase the risk awareness of the workers, or improves the revival of safety knowledge.

The SSS at the level 2 are able to facilitate workers to apply operating instructions or procedures extremely important for preventing a major accident.

The level 3 corresponds to the smart systems relevant to the safety in process activities. They provide data on equipment, materials and other safety relevant resources, but also control the loss of containment, in terms of energy or hazardous materials, for avoiding a major accident.

The high level, value 4, is able to manage the data providing the monitoring of equipment, substances and other safety resources, with the aim to prevent the loss of containment. The SSS at this level are able to reduce uncertainties on the accident likelihood evaluation.

\subsection{Effectiveness criteria}

This criterion deals with the needs to estimate more expected the likelihood of accident, which is a critical issue for all Seveso practitioners. They have to trust in generic failure rates, which, by definition, are based on averaged data coming from a large number of facilities using different technical and organizational systems.

Pitblado et al. [2], at the end of a detailed review of the methods to quantify the expected reduction of accident frequency resulting from the adoption of enhanced process safety management practices and technical safety systems, affirmed that the bow tie approach is the only method able to generate believable modification factors. The risk analyst may include any barrier affecting the accidental chain (preventive or protective; technical or procedural) in the bow tie schema, evaluating its likelihood of failure, which in turn affects the likelihood of major accident.

In such a way, the analyst should deal even with a new SSS installed to control a specific hazard. It could be a further barrier to prevent a loss of containment of hazardous materials or to avoid severe consequences in the event of an uncontrolled leak. The probability of failure of the SSS or, if available, its Safety Integrity Level SIL will be accounted to update and, hopefully, decrease the likelihood of major accident.

If this procedure is applicable, the effectiveness of the new SSS is easy to estimate, as the approach is the same as for SIL. That is perfect for the SSS aiming at the prevention of the loss of containment of either energy or hazardous materials. 
Table 2: Level of relevance.

\begin{tabular}{|l|l|}
\hline Level & Requirements \\
\hline 1 & Production - Quality \\
\hline 2 & Health \& Safety of Work Environment \\
\hline 3 & Safety of Industrial Processes \\
\hline 4 & Prevention of Major Accident \\
\hline
\end{tabular}

Unfortunately, not all SSS work in this way. As discussed in the previous paragraph, there are a few SSS, which should be classified at a lower "adequateness level" as they are aiming at occupational safety and positive indirect effects are expected for the control of major accident hazard. In this case, the bow tie is difficult to use, as it is not clear the specific hazard to put at the center. Thus, we had to adopt a much rougher approach, using the deprecated correction factors. Indeed, the correction factors leave room for subjective aspects, they are unbalanced and easily questionable; but there is no better and the analyst has to use them, even reluctantly.

A few very simple correction factors may be found in AMINAL [3], which is a trusted information base for safety reports writing. AMINAL has factors for both organizational and technical measures (simple or redundant). A more complete and detailed source may be found in API 581 [4]. It includes a detailed and updated system for scoring SMS. The score, even designed for the likelihood of equipment failure, has been forced, in this paper, for the likelihood of loss of containment. API 581 assigns different weights to the points in the SMS, thus we can understand how much that point may be relevant to major accidents. AMINAL values are versatile indeed, but quite vague and too optimistic; API581 are much sharper but they are provided just for systems dealing with equipment integrity and functionality, as chapter 5 will discuss in detail.

The Table 3 shows the levels adopted, which correspond to different factors as result of the integration of diverse studies. Indeed, the factors outcome from the combination of AMINAL, tempered by the authors' judgement, and API581 values.

The level 1 corresponds to generic improvements. The level 2 deals with improvements of more specific to procedural and organizational barriers. Both the level 3 and 4 indicate an increase of the technical safety measure, but the level 4 foresees a higher reliability factor, where the SSS implementation is redundant in design phase and their monitoring is active in service.

Table 3: Level of effectiveness.

\begin{tabular}{|l|l|l|}
\hline Level & Factor & Requirements \\
\hline 1 & $0.9-0.5$ & Generic improvements \\
\hline 2 & $0.5-0.1$ & $\begin{array}{l}\text { Procedural and/or Organizational } \\
\text { improvements }\end{array}$ \\
\hline 3 & $0.1-0.05$ & Technical safety measure \\
\hline 4 & $0.05-0.01$ & $\begin{array}{l}\text { Technical safety measure with a higher } \\
\text { reliability level }\end{array}$ \\
\hline
\end{tabular}




\section{SMART SAFETY SYSTEMS (A FEW EXAMPLES)}

Fig. 1 graphically illustrates the features commonly used to characterize a smart system, including: sensor to detect data, identification, wireless communication, capability of machine to machine M2M transmission, and the software for managing all of them. Indeed, the software is the key feature able to upgrade a smart technology into a smart system, transforming data into information and, hopefully, into knowledge.

The software implements the functions and specializes the new technology into a particular industrial application context for meeting specific needs.

Another key aspect of introducing smart technologies is the wireless network of smart systems, which is typical of IoT technologies. The characteristics of the adopted network influences the improvement of the capabilities of M2M communications, and the management of data and information. Indeed, the network should be flexible and scalable to be extended, reduced, or integrated with existing wired networks.

Here is the discussion of a few representative examples of smart systems for safety purposes. They are grouped, based on the application and the target, including monitoring asset, supporting operations, protecting workers, working in emergency.

Each example illustrates the adopted smart technologies, focusing on the details only when they are particular innovative or no popular for a common use.

\subsection{Monitoring asset}

Monitoring means observe, check, maintain a regular surveillance, in this context over the asset of an establishment. It is already a well-known concept in the process control, where wired or wireless systems constantly control the physical parameters, including: pressure, temperature, flows, and level. However, here monitoring means checking and detecting data related to phenomena deterioration that may cause asset failure.

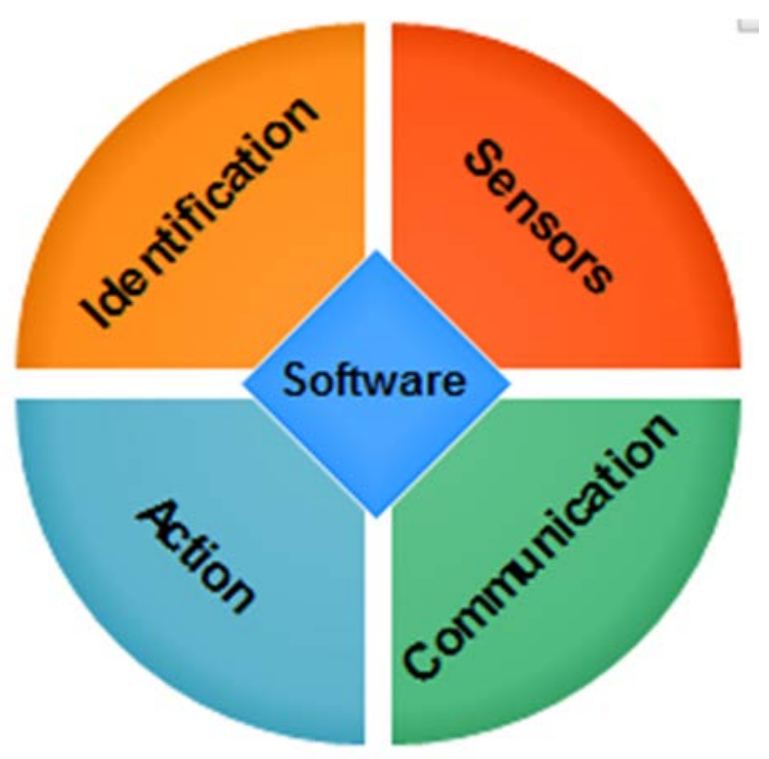

Figure 1: Graphical representation of smart system for safety. 


\subsubsection{Erosion}

The problem of erosion in oil \& gas establishments is today growing due both to the characteristics of crude treated and to the carbon steel pipelines adopted for cost saving reasons but vulnerable to saline formation water. Wold et al. [5], discuss about non-intrusive, Sand Monitoring Technologies based on Acoustic sensors. Those systems measure acoustic impacts and noise, and the amount of particles in water, oil, gas.

The solutions are already commercial, installed in offshore and refineries. Thus, the reliability of the technology is high, and it is reasonable to think that the SMS adopted are certified, but are also able to manage the information coming from those data. Referring to the Readiness Table 1, the level assigned to this SSS should be almost 4 . The system is able to check the sand content, but it does not refer directly to the pipelines or asset failures, therefore the relevance assigned is level 3. On the other hand, this system can be adopted as technical measure for prevention, so that the effectiveness level is 4 .

\subsubsection{Corrosion}

The most useful effect of corrosion and erosion phenomena is the thinning. The thickness of pressure equipment, including pipework, reactors and heat exchangers, must be periodically inspected, in order to prevent wall impairment and consequent loss of containment and to forecast the remaining useful life of the equipment. There are many techniques suitable for these measurements and some of them are usable for continuous monitoring.

The Ultrasound Testing UT is one of the most flexible, versatile and promising technique. The SS based on UT technology, basically, includes a high-frequency ultrasonic emitter, a sensor, a tag or a locator device and a wireless communication system. A specialized software directly analyzes the response of the signal sent directly into the equipment. Thus, by the time difference and the ultrasonic wave velocity, it is possible to compute the thickness of pipe or vessel wall. The relevance level assigned is 4, according to the Table 3 .

Adopting this type of monitoring allows having more time for appropriate actions, and may reduce the likelihood of major failures ruptures. The effectiveness assigned to this type of SSS is equal to 3.

With regard the readiness of this SSS, the level assigned is 4 , if included in a "smart" SMS.

\subsubsection{Defects}

For a couple of decade, Acoustic Emission technology has been used for detecting and locating defects (e.g., micro crack) in structures and components, both in industrial and civil engineering.

In the European Project Industrial Safety and Life Cycle Engineering IRIS [6], an AE-based application has been developed to monitor hot pipes at a power plant in operation. The system is compound by a digital signal processor, standardized data transfer ports, reconfigurable logic and specific software.

The wireless sensor based on AE technology requires worthy of precision in finding the correct position of possible defect. Mennuti et al. [7], describe a few localization techniques with possible hardware implementations.

All these solutions correspond to prototypes whose value is examined in laboratory, but more research and test cases are needed. The relevance and the potentiality of their effectiveness are both at level 4. The readiness of this type of SSS is at most 2. 


\subsubsection{Noise/vibration}

In the process plants, there are a lot of rotating machineries, including pumps, compressors, which are critical for major accidents. At refineries, they are usually redundant and are checked manually periodically for vibration.

Schodowski [8], illustrates how a Wireless Monitoring of pumps' Vibration, coupled with adequate software, allows early detection of anomalies and a risk reduction. Belanger and Sprayberry [9] describes a case study of the installation of wireless vibration transmitters at the pumps of a refinery. This system is not only a noise detector, but it checks for a "special noise", something that points out a different type of vibration, as a precursor of possible damage.

Some wireless vibration sensors are already available on the market and adopted in the refineries, so that the technology readiness is at level 4 , if coupled with a smart SMS. The system can also be considered as a technical barrier to prevent major accident, reducing its likelihood, so far, the relevance is at level 4. The level of effectiveness is at value 3 .

\subsubsection{Identification of critical equipment}

In chemical establishment, Critical Equipment Identification is an important task. Equipment, considered critical for major accident, has to be marked, for example, by visible signals.

QR codes and RFID (Radio Frequency Identification) are well-known techniques adopted for persons and objects identification. Those smart identification systems are frequently used in daily life-places, while they are still new inside Seveso establishment, requiring further customization.

These systems contribute to improve the knowledge of operators and workers; their relevance is assigned to level 3. They have also a positive impact in the organizational management, so that the effectiveness level is 3 . Their readiness is high and can be integrated into traditional SMS, therefore the level assigned is 3.

\subsubsection{Gas detection}

Wireless Gas Detection systems consists of multiple sensors communicating into a network to a control room, and of alarms for warning the presence of gas escape. There are also available in wearable configuration, suitable for personal protection. Thus, they are appropriate for occupational health and safety protection, but can be useful for mitigating the consequences of toxic gas major release.

Those kinds of systems are already commercially available, and adopted in mature chemical industries, so that their readiness level is 4 , if included in a smart SMS. Their relevance and their effectiveness are both at level 3.

\subsection{Supporting operations}

The examples described below show smart systems designed for making some industrial operations safer.

\subsubsection{Transfer operations by crane}

Cranes are well-known critical equipment for constructions, but bridged cranes and mobile cranes are also widely used in process industries. Thus, crane operations may trigger accident involving hazardous materials, so that crane's risks are relevant in Seveso establishments, as detailing illustrated by Milazzo et al. [10].

SPRINCE project (Smart PRocess INdustry CranEs) objective is the prevention of crane accidents due to obstructed view, as discussed by Ancione et al. [11]. The project aims to 
develop an augmented-vision system able to prevent any type of collision, with people or objects. The system is compound of a couple of web-cameras strategically mounted for avoiding interferences of load with obstacles. A specific software, based on stereoscopic images algorithms and statistical models, supports the technology for providing the operators with real-time solutions and appropriate alert signals.

The project results have been validated in the lab, and tested in case study to a port area, but this solution requires some improvement before their full adoption in an industrial environment. So far, the readiness level assigned is 2 at most. The relevance of this system is at level 3. SPRINCE has major impact on human factors, since it gave remarkable advantages to the crane operators, but also to safe conditions in the working area and during transfer activities. Thus, its effectiveness is at level 3.

\subsubsection{Transfer operations by forklift}

In the risk analysis of chemical establishment, many top events deal with forklift operations, and the number of near misses, dangerous situations or incidents involving goods movements is remarkable, too.

An application of Real Time Location System RLTS, based of RFID technologies, has been developed by Bragatto et al. [12]. The goal of the system is to prevent collisions during the movement in stock keeping departments with hazard products, where the traffic is high and pedestrian and forklifts work closely.

Some commercial forklift, e.g., Toyota Safety System, already have these smart sensors on board. Thus, the technological readiness is high, at level 4, if included into a smart SMS. In chemical establishments, the risk analysis points out many top events related to forklifts operations, and the number of dangerous situations is important, so that the relevance of this solution is level 4 . The system is a technical safety prevention measure; therefore, its effectiveness is at level 4.

\subsection{Protecting workers}

The IoT technologies is widely used for improving Personal Protective Equipment PPE, as illustrated by Podgòrski et al. [13]. Below some examples more related to Seveso establishments are described.

\subsubsection{Position identification of workers}

There are many systems for tracing the workers' presence inside an establishment. They are tags for identifying workers, people location and position in the working areas. Even though they are aiming at optimizing personnel organization, they have a few potential side effects on safety management, for instance, to collect and record near misses.

The relevance of the Position Smart Identification system is at level 1, since they mainly increase the awareness of the workers. The effectiveness is a generic improvement, so that at level 1. The readiness is high, at level 4, if included in a smart SMS.

\subsubsection{Systems for using PPE}

The project SODYRA (Smart Object for Dynamic Risk Analysis), designed by Gnoni et al. [14], addresses the problem related to the use of PPE's on the basis of worker's profile, task and type of machinery handled. It aims to support an effective knowledge management about risks and workplace current conditions. Two main hazardous situations are examined, the risks due to an incorrect use of PPE and the risks due to collision accidents at workplace. The system integrates several basic smart technologies (e.g. beacon system, Bluetooth Low 
Energy BLE), and it will be worn by a worker before accessing the workplace and during its shift.

This solution supports workers in applying procedures and operative instructions on the appropriate use of PPE. It improves the occupational safety, but potentially prevents the accidents triggered by the manual handling of hazardous materials. The relevance level is 3 , and the effectiveness is 3 . SODYRA has already been tested in a pilot plant; therefore, its readiness is at level 3.

\subsubsection{Eye wash system}

An emergency Eye Wash/Shower could be placed near equipment containing hazardous materials, which might spray on worker's face or body. Those devices with wireless connection are SSS providing an alarm directly to the control room when they are used. The alarm activation denotes an undesired event occurred, therefore an assistance or investigation can be quickly activated.

The system is already commercially available and adopted in several chemical establishments. For this reason, its readiness is at level 4, if included in a smart SMS. The system indirectly detects the leakage of hazardous substances, and increases the awareness of the worker therefore its relevance is at level 1. Since it works as a near-miss detector, it generally improves the safety conditions, so that its effectiveness is at level 1.

\subsection{Working in emergency}

In this research, the working in emergency means the activities done by the emergency team internal to the establishments.

\subsubsection{Systems for hazard awareness}

In rescue missions, the situational awareness is a key factor for emergency activities operating in congested indoor environment. The RISING (indooR localizatIon and building maintenance using radio frequency Identification and inertial NaviGation) project's goal is to support operators providing them on field with a system for situational awareness and personnel positioning [15]. The RISING solution integrates RFID (Radio Frequency Identification) with the inertial navigation.

This solution is a technical measure for mitigating the consequences in case of accidents or incidents; its relevance is at level 4. It has been tested in a pilot case; therefore, its readiness is at level 2. The assigned level of effectiveness is 3 .

\subsection{Classification of the proposed SSSs}

The Table 4 summarizes the examples of SSS previously described, referring to which level is assigned, for all criteria that are readiness (Rd), relevance (R), and effectiveness (E).

Table 4: Classification of SSS with respect to criteria.

\begin{tabular}{|l|l|l|l|}
\hline Smart Safety System & Rd & R & E \\
\hline Sand Acoustic Monitoring & 4 & 3 & 4 \\
\hline UT thickness monitoring & 4 & 4 & 3 \\
\hline Acoustic Emission for Defect Detection & 2 & 4 & 4 \\
\hline Wireless monitoring vibration & 4 & 4 & 3 \\
\hline
\end{tabular}


Table 4: Continued.

\begin{tabular}{|l|l|l|l|}
\hline Smart Safety System & Rd & $\mathrm{R}$ & $\mathrm{E}$ \\
\hline Critical Equipment Identification & 3 & 3 & 3 \\
\hline Wireless Gas Detection & 3 & 3 & 4 \\
\hline SPRINCE - operations by cranes & 2 & 3 & 3 \\
\hline Toyota safety system - by forklift & 4 & 4 & 4 \\
\hline Position Smart Identification & 4 & 1 & 1 \\
\hline SODYRA for PPE use & 3 & 3 & 3 \\
\hline Eye wash/shower & 4 & 1 & 1 \\
\hline RISING for emergency & 2 & 4 & 3 \\
\hline
\end{tabular}

Table 5: SSS integration into SMS.

\begin{tabular}{|l|l|}
\hline Smart Safety System & SMS keys \\
\hline Sand Acoustic Monitoring & Operating control \\
\hline UT thickness monitoring & Operating Control \\
\hline Acoustic Emission for Defect Detection & Operating Control \\
\hline Wireless monitoring vibration & Operating Control \\
\hline Critical Equipment Identification & Hazard Identification \\
\hline Wireless Gas Detection & Hazard Identification \\
\hline SPRINCE - operations by cranes & Operating Control \\
\hline Toyota safety system - by forklift & Operating Control \\
\hline Position Smart Identification & Resources \\
\hline SODYRA for PPE use & Training \\
\hline Eye wash/shower & Near Misses \\
\hline RISING for emergency & Emergency \\
\hline
\end{tabular}

\section{MANAGEMENT OF SMART SAFETY SYSTEMS}

As said in $\S 2$ an SSS may be useful for safety purpose just if it is integrated in the SMS, which, through defined procedures and instructions, connects resources data, documents, information, experience, and knowledge. If a SSS is not canalized into the SMS, it is simply a "gimmick". For that reason, the first step to evaluate SSS candidate to be adopted is to compare it with the SMS and find where it may be plugged. All SMS are based on the well-known Deming cycle: Plan Do Check Act. In the detail, this basic schema may be implemented in slightly different ways. This paper uses the schema proposed by the Italian Standard Organization for the Seveso plant, which deals Hazard Identification (PLAN); Resources, Training, Documents, Operating Control, Emergency, Management of Change, (DO); Surveillance, Incidents \& Near Misses, Internal Audit (CHECK); Review (ACT).

Table 5 shows how the SSS described in section 4 may be integrated into the SMS.

\section{CONCLUSION}

Seveso establishments require special attention, even when improvements are introduced. The decision makers should not be enthusiastic about technology but understand what can really be useful in preventing major accidents. The paper provides the first attempt to define an evaluation grid, for helping decisions on this subject. As discussed in the paper, quantitative risk assessment is already possible and can be the basis for assessing the cost and benefits of investment in safety. 


\section{REFERENCES}

[1] Bragatto, P.A., Agnello, P., Ansaldi, S.M. \& Pirone, A., Improved safety procedures for small chemical companies. Safety and Reliability of Complex Engineered Systems, Taylor \& Francis Group, London, 2015.

[2] Pitblado, R., Bain, B., Falck, A., Litland, K. \& Spitzenberger, C., Frequency data and modification factors used in QRA studies. Journal of Loss Prevention, Elsevier, 24, pp. 249-258, 2011.

[3] Flemish Government, AMINAL Handbook failure frequencies for drawing up a safety report. LNE Department BE, 2009.

[4] API 581. Risk-based inspection methodology, API RP 581. American Petroleum Institute, 3, 2016.

[5] Wold, K., Stoen, R. \& Rapone, M., Integration of intrusive and non-intrusive methods for corrosion and sand/erosion monitoring. La Metallurgia Italiana, 2, 2012.

[6] Wenzel, H., IRIS - Industrial Safety and Life Cycle Engineering: Technology, Standards, Applications. VCE Vienna Consulting Engineers ZT GmbH, Vienna, pp. 293-303, 2013.

[7] Mennuti, C., Augugliaro, G., De Petris, C., Cardarilli, G., Di Nunzio, L. \& Fazzolari, R., Tecniche per la localizzazione di danni strutturali per mezzo di AE: Algoritmi e possibili soluzioni HW per WSN. SAFAP Conference, Milano (in Italian), 2016.

[8] Schodowski, E., Wireless sensing improves refinery operations. American Fuel \& Petrochemical Manufacturers, 2016.

[9] Belanger D. \& Sprayberry J., Streamline API 691 Compliance and Reduce Automation Risks Simultaneously. White Paper, Process System \& Solution, Emerson Process Management, www.emersonprocess.com. Accessed on: Nov. 2016.

[10] Milazzo, M.F., Ancione, G., Spasojevic Brkic, V. \& Vališ, D., Investigation of crane operation safety by analyzing main accident causes. Risk, Reliability and Safety: Innovating Theory and Practice, Taylor \& Francis Group, London, 2017.

[11] Ancione, G., Kavasidis, I., Merlino, G. \& Milazzo, M.F., Real-time guidance system for cranes to manage risks due to releases of hazardous materials. Risk, Reliability and Safety: Innovating Theory and Practice, Taylor \& Francis Group, London, 2017.

[12] Bragatto, P.A., Pirone, A. \& Gnoni, M.G., Application of RFID technology for supporting effective risk management in chemical warehouses. Safety, Reliability and Risk Analysis: Beyond the Horizon, Taylor \& Francis Group, London, 2014.

[13] Podgòrski, D., Majchrzycka, K., Dąbrowska, A., Gralewicz, G. \& Okrasa, M., Towards a conceptual framework of OSH risk management in smart working environments based on smart PPE, ambient intelligence and the Internet of Things technologies. International Journal of Occupational Safety and Ergonomics (JOSE), 23(1), pp. 1-20, 2017.

[14] Gnoni, M.G., Elia, V. \& Bragatto, P.A., An IOT based system to prevent injuries in assembly line production systems. IEEE International Conference on Industrial Engineering and Engineering Management (IEEM). Bali, Indonesia, pp. 1889-1892, 2016.

[15] De Cillis, F., Inderst, F., Pascucci, F., Setola, R., Tesei, M. \& Bragatto, P.A., Improving the safety and the operational efficiency of emergency operators via onfield situational awareness. Chemical Engineering Transactions, 53, pp. 331-336, 2016. 